\title{
Characteristics of the Turbulence Processes in the Magnetohydrodynamic Environment
}

\author{
By Liudmyla Kozak* \\ Bogdan Petrenko ${ }^{\dagger}$ \\ Elena Kronberg ${ }^{*}$ \\ Elena Grigorenko ${ }^{+}$ \\ Antony Lui ${ }^{\star}$
}

\begin{abstract}
Methods and approaches that can be used for analyzing the hydrodynamic and magnetohydrodynamic turbulent flows are proposed. It is shown that the best methods to characterize the types of turbulent processes are the methods of statistical physics. Within the statistical approach the fractal analysis (determination of the fractal length and height of the maximum of the probability density fluctuations of the studied parameters) and the multifractal analysis (study of a power dependence of high order statistical moments and construction multifractal spectrum) had been carried out. It is indicate that the statistical analysis of properties of turbulent processes can be supplemented by the spectral studies: Fourier and wavelet analysis. In order to test the methods and approaches we have used the magnetic field measurements from the space mission Cluster-II with a sampling frequency of $22.5 \mathrm{~Hz}$ in the Earth's magnetospheric tail. A good agreement between the investigations and the mutual additions to provide an overall view of the turbulence can be noted.
\end{abstract}

Keywords: Fourier and wavelet analysis, Fractal and multifractal analysis, Kolmogorov and electron-magneto-hydrodynamics model of turbulent processes, Turbulence spectra in the tail of the Earth's magnetosphere, Turbulent processes.

\section{Introduction}

Turbulence is the natural state of the hydrodynamic flows and cosmic plasma; therefore, studying its characteristics is essential for the understanding of the fundamental properties of nature. In magnetohydrodynamics, the properties of turbulence can be dramatically affected both by flow boundaries and the scales of the structures (waves, vortices, etc.) formed by magnetic and electric fields. The turbulence of plasma flows can be generated by many classes of instabilities: drift dissipative, kinetic, magnetohydrodynamic, etc. In addition, turbulence is

\footnotetext{
*Associate Professor, Kyiv Taras Shevchenko University and Space Research Institute, National Academy of Sciences of Ukraine and State Space Agency of Ukraine, Ukraine.

${ }^{\dagger}$ Student, Kyiv Taras Shevchenko University, Ukraine.

${ }^{*}$ Researcher, Max Planck Institutefor Solar System Research and Ludwig Maximilian University of Munich, Germany.

${ }^{+}$Senior Researcher, Space Research Institute, RAS, Russia.

•Principal Professional Staff, Johns Hopkins University Applied Physics Laboratory, USA.
} 
characterized by a large number of degrees of freedom and nonlinearly interacting modes. Scientists typically use statistical physics and the theory of probability to describe such a medium. This way they can obtain information about average variations in the macroscopic parameters of the plasma medium in time (or space) without scrutinizing the conditions of excitation of specific nonlinear processes.

Despite more than fifty years of research and many significant results, the satisfactory theory of MHD turbulence remains difficult to access. Indeed, even the simplest (most idealized) cases are still not fully understood.Some of them are listed below:

How is turbulence amplified, supported and determines the shape of magnetic fields? What are the structure and spectrum of these fields on different scales? (Due to this, the turbulence is directly related to the fundamental problem of magnetic field generation).

How is energy transferred between cascades and how is it dissipated? (In accretion disc and the solar corona, for example, it would be important to know if plasma heating is due to the turbulent processes).

How the turbulent plasma flow and the magnetic field amplify or restrain the heat transfer.

This is far from a complete list of questions that arise in the analysis of space and laboratory plasma.

Usually, the framework of the study does not provide answers to all posed problems, but significantly can help in understanding the pattern of turbulent and self-organized processes in complex systems, including plasma and magnetic field.

From previous studies, it is known that most astrophysical plasmas are collisionless at kinetic scales, so that Solar System may be considered as a 'laboratory' for plasma physics. In laboratory plasmas, kinetic scales are typically of the order of a few centimeters or less unlike in the near-Earth space, where high resolution only in situ measurements allow studying turbulent energy dissipation under different conditions over an enough large range of scales. Due to similarities with various kinds of plasmas regimes, many of the results obtained in near-Earth space in virtue of space mission measurements are advantageous for understanding other plasma environments (THOR, 2017; Grigorenko et al., 2016; 2018; Malykhin et al., 2018).

The characteristic of plasma turbulence is not only the kinetic number of Reynolds, Re, which is determined by the kinematic viscosity, but also the Reynolds magnetic number, Rem, which is related to the magnetic viscosity. For relatively low values $\operatorname{Re}$, Rem $<10^{3}-10^{4}$, and under the influence of boundaries in the plasma, the feature of intermittence (heterogeneity) can be formed, which means mutual coexistence of the active regions with passive ones where the flow is quasi-laminar (Novikov and Stewart, 1964). In this process, the plasma parameters are observed randomly with the non-Gaussian distribution function, thus, the large amplitude turbulent pulses are presented in the process with a probability much greater than that, assumed by the Gaussian law (normal 
distribution).The intermittency is observed in hydrodynamic turbulent flows within neutral media (Zaslavskyand Sagdeev, 1988) and magnetized plasma (see, for example, (Frisch, 1998), etc), both with large and moderate Reynolds numbers $(<1000)$.

The peculiarities of the intermittency in the plasma boundary layers are the presence of magnetic and electric fields, which lead to additional anisotropy of the process (Kozak et al., 2015). In the central regions of the plasma medium, where the influence of the boundary layers is insignificant, the properties of plasma turbulence can vary considerably.

In the turbulent medium, the intermittence is observed in the form of large amplitude pulsations (Frik, 1999). Laws of scale similarity (scaling) of such intermittent turbulence are described by parameters that are scale-dependent (multiscale). From the theoretical consideration it follows, that the property of the turbulence intermittency is related to the hidden statistical symmetries (symmetries of scale invariance) of the dynamic equations describing the motion, and the need to establish a large-scale invariance in a bounded space of the transition region (Frik, 1999). Random pulsations of velocity and other parameters of a turbulent flow with intermittence have non-Gaussian statistics, that is, they are not described by the classical (normal) diffusion law. In terms of the most general theoretical concepts, dynamics of such a process can be described by the power laws of distribution, multiscale, i.e. a spectrum of characteristic scales. Analytical and numerical solution to the problem of the turbulent plasma dynamics (in three-dimensional geometry) and scaling turbulence determination with the necessary resolution on large timescales are currently not possible. Since developed turbulence is characterized by a large number of degrees of freedom and nonlinear modeinteractions, a multiscale structure and random pulsations of velocities and fields, methods of statistical physics and probability theory are best suited for the turbulent medium description. In order to describe a random process, it is necessary to know the distribution function of amplitudes of MHD parameter fluctuations.

The probability distribution function of such processes is not always described by known mathematical functions and series; therefore for many types of random processes only the approximation method of their distribution functions is applicable.

Often, the probability distribution functions of fluctuations satisfy the Gaussian distribution (normal law). Gaussian statistics, for example, satisfy the classical Brownian motion. Other likelihood probabilities are also known in probability theory, which are capable of describing random processes with longrange correlations. The statistical description is associated with some information coarse-grain procedure and reduces the number of variables in the task. At the same time, a significant part of the information about the state of individual particles (or waves) is lost, but sufficient information is provided about the macroscopic nature of the motion and the probable distribution of the system states. Therefore, it is necessary to apply special methods and approaches for turbulence description. 
Since for physical applications important properties of nonlinearity, heterogeneity and anisotropy of the turbulent process are important, the moments of the distribution function are then investigated, which allows us to approximately describe most of the main effects.

Consequently, it is necessary to determine from the experiment the statistical properties of turbulence associated with the large-scale invariance, and obtain estimates for scaling (indicators of the degree of dependence of plasma parameters in the assumption of power laws). This gives an opportunity not only qualitatively and quantitatively describe the features of turbulent processes, but also to understand the properties of the transport in the considered ranges.

\section{Used Methods and Approaches}

Statistical Analysis

\section{Analysis of the Probability Distribution Function of Fluctuations}

The probability distribution function (PDF) of the fluctuation amplitudes of the simplest random process, as noted in the introduction, is the Gaussian law (Consolini and Lui, 2000; Consolini et al., 2005; Kozak et al., 2012; Kozak et al., 2015). From the behavior of the probability distribution function (PDF), one can determine the characteristic spatial or temporal scale, which is determined by the step at which the PDF loses the gaussian property. In this case, in the presence of heterogeneity of turbulent processes, we observe a significant "widening" of PDF. The probability of significant fluctuations on the distribution tails will be high due to the excess energy of large-scale perturbations generated by the source.

\section{Analysis of the Moments of the Distribution Function}

In the analysis of the fluctuations of the MHD parameters $(\mathrm{X}(\mathrm{t}))$, the moment of the distribution function (structure function) Sq of the order $\mathrm{q}$ is determined by the scale $\tau$ as the statistical mean of the relation ensemble $\delta \tau X=X(t+\tau)-X(t)$, and $\mathrm{Sq}(\tau)=\left\langle|\delta \tau \mathrm{X}|^{\mathrm{q}}\right\rangle$. In the Kolmogorov theory, the structure functions (moments) for the speed difference on a spatial scale 1 are considered: $\delta l v=v(x+1)-v(x)$, $\operatorname{Sq}(1) \equiv\left\langle|\delta l v|^{\mathrm{q}}\right\rangle$.

The study of structure functions is equivalent to the PDF study of turbulent fluctuations. From a practical point of view, it is easier to explore structure functions, in most cases they can be measured in an experiment. The method of structure functions allows us to describe in detail the heterogeneity of the distribution at different scales of the process. For an isotropically developed turbulence, Kolmogorov considered the turbulent cascade and suggested that in the inertial interval $\eta<<1<<\mathrm{L}$ ( $\eta$ is dissipation scale, and $\mathrm{L}$ is a global scale) for large Reynolds numbers, all statistically averaged moments $\mathrm{Sq}(\mathrm{l})$ of velocity fields $v$ on a scale 1 depend only on the average dissipation ratecl and the given scale 
1 (property of the locality). This theory by Kolmogorov was named K41 (Kolmogorov, 1941). In the inertial range, the theory K41 supposes statistical quasi-equilibrium of fluctuations and Gaussian statistics of velocity pulsations. Dynamics in the inertial range does not depend on the method of turbulence generation and is determined by the energy flux invariant through this interval: the average energy flow remains (Chang, 1992).

This leads to the fact that the expression for the structural function (statistical mean) is related to the spatial scale of the power law (Barenblatt, 2004; Kolmogorov, 1941): $\operatorname{Sq}(1) \sim\left\langle|\delta 1 v|^{q}\right\rangle \equiv\left\langle|v(x+1)-v(x)|^{q}\right\rangle \sim 1^{\zeta(q)}$.

From the dimensional analysis, Kolmogorov derived the famous power law for the energy spectrum Ek $\mathrm{k}^{-5 / 3}$ (the law of the "fifth thirds") for the developed isotropic turbulence in the inertial range). In the K41 model, the scaling of structure functions is linear $\zeta(q)=q / 3$, which reflects the fact that turbulent processes are uniform. For heterogeneous turbulent processes, highorder structure functions have a nonlinear dependence $\zeta(\mathrm{q})$ on order, which reflects the fact that PDF deviates from the Gaussian law.

In the Kolmogorov model $\mathrm{K} 41$, for the inertial range, there are four assumptions: homogeneity (no singular points), large-scale invariance (no special scales), isotropy (no special directions) and locality of interactions (the interactions between comparable scales dominate). The local nature of turbulence in the inertial range leads to energy changing on a given scale is determined by the interaction of only vortices with similar values of wave numbers and a long time (more than the eddy turnover time). Interaction of vortices with very different sizes is relatively weak.

The presence of magnetic fields in the interplanetary medium can cause a strong anisotropy of the dynamic processes in the plasma. An attempt to adapt the model K41 to a magnetized medium is the two-dimensional model of IroshnikovKraichnan (IK) (Kraichnan, 1959; 1970). Within this framework, turbulent disturbances are small in amplitude and similar to wave disturbances propagating along the middle field (Alfven turbulence). The energy spectrum for the IK model is given by the equation: $\operatorname{EIK}(\mathrm{k})=\left\langle|\delta v(\mathrm{k})|^{2}\right\rangle \mathrm{k}^{2} \propto \mathrm{k}^{-3 / 2}$. In this case, in comparison with the Kolmogorov spectrum, the level of energy transport on small scales is significantly reduced, and the time of energy transport increases. Dependenceon structure functions in the IK model: $\mathrm{Sq}(\mathrm{l}) \mathrm{l}^{\mathrm{q} / 4}$.

In the Kraichnan approach, the influence of large-scale fluctuations on the evolution of small-scale in homogenities is exaggerated: this effect is reduced to the transfer of small-scale fluctuations with a small deformation (adiabatic approximation) (Kraichnan, 1959). Despite the drawbacks, the IK model is widely used in many works (for example, to interpret the properties of turbulence of interplanetary plasma, plasma in tokamaks).

For analysis of the distribution function moments, the presence of the property of extended self-similiraty is decisive. This phenomenon was discovered experimentally in the study of small-scale hydrodynamic turbulence in wind tunnel (Benzi et al., 1993). At relatively low values of the Reynolds numbers, when in the usual representation $\mathrm{Sq}(\mathrm{l}) \sim 1^{\zeta(\mathrm{q})}$ the inertial interval is not detected, there is a dependence: $\mathrm{Sq}(1) \sim \mathrm{S} 3(1)^{\zeta(\mathrm{q}) / \zeta(3)}$ for an extended range of scales $1 \geq$ 
$5 \eta$, where is $\eta$ is the Kolmogorov scale of dissipation. This property (also called self-similarity) is observed almost to the extent of dissipation.

This phenomenological observation led to the development of a criterion for generalized self-similarity for an arbitrary pair of structure functions $\mathrm{Sq}(\mathrm{l}) \sim$ $\operatorname{Sp}(1) \zeta(\mathrm{q}) / \zeta(\mathrm{p})$.

It is assumed that this self-similarity is a manifestation of hidden statistical symmetries.

Using the property of extended self-similarity, one can find with a fairly good accuracy the value of $\zeta(q)$ and estimate the type of turbulent processes. In this case, the nonlinear functional dependence $\zeta(q)$ of the momentorder $q$ for experimental data, as indicated above, is the result of the intermittency of the process. For the interpretation of the nonlinear spectrum $\zeta(q)$, the log-Poisson model of turbulence is used, in which the power index of the structure function is determined by the relation (Dubrulle,1994; Kozak et al., 2011; She and Leveque, 1994):

$$
\zeta(q)=(1-\Delta) \frac{q}{3}+\frac{\Delta}{1-\beta}\left[1-\beta^{q / 3}\right]
$$

where, $\beta$ and $\Delta$ are parameters that characterize the intermittency of the process and the form of dissipative structures, respectively. It is important that within the framework of this model the stochastic multiplicative cascade is considered, and the logarithm of dissipation energy is described by the Poisson distribution. For isotropic three-dimensional turbulence, She and Leveque proposed $\Delta=\beta=2$ / 3 (She and Leveque, 1994).

It should be noted the practical usage of log-Poisson models for the interpretation of experimental data. The advantage of the log-Poisson model is to take into account the influence of the dissipative range when the boundary effects with a limited inertial range play a significant role in the system with a relatively small value of Reynolds number. The property of generalized self-similarity takes into account boundary effects: large-scale invariance is not formed in the infinite space, but at the finite interval of scales. It naturally allows on to analyze the effects of viscosity (dissipation) and properties of dissipative structures, in particular, their dimension. For example, in the log-Poisson model of isotropic three-dimensional hydrodynamic turbulence it is assumed that the onedimensional filamentous structures correspond to the dissipation. In the logPoisson model, taking into account the two-dimensional empirical model of the Iroshnikov-Kraichnan model, two-dimensional dissipative structures are assumed (Boldyrev, 2006; Dubrulle, 1994; Gledzer, 2005). If the medium is characterized by complex geometry of dissipative structures or at the same time there are structures with different dimensionality, then the process can be characterized by the values of the fitting $\beta$ and $\Delta$. 


\section{Spectral Analysis}

Turbulent processes are characterized by a system of vortex hierarchy, in which there is a cascade of energy from large scales, comparable to the size of the source, through the inertial range, to small dissipative scales. The coarsest characteristic of the turbulent medium is the spectral index, which characterizes the change in spectral density of power in the inertial range.

The inertial interval with a power spectrum is limited by low frequencies associated with large-scale energy sources and frequencies that correspond to small-scale dissipative (kinetic) processes. The dynamics of the inertial interval does not depend on the turbulence excitation mechanism and is also determined by the invariant of the energy flux through this interval as mentioned above.

The main assumption for statistical (multifractal) analysis is that the signal we are considering is stationary, that is, the characteristic times do not change within the considered interval. However, for a very dynamic situation, the stationary signal cannot be excluded. Therefore, spectral and wavelet analysis was conducted.

\section{$\underline{\text { Wavelet Analysis }}$}

In the framework of the wavelet analysis, for the series of measurements (time series, $x_{n}$, with equal time spacing $\delta t$ and $n=0 \ldots N-1$ ), we used Morlet wavelet, which consists of a plane wave modulated by a Gaussian:

$\psi_{0}(\eta)=\pi^{-1 / 4} e^{i \omega_{0} \eta} e^{-\eta^{2} / 2}$

where $\omega_{0}$ is the non dimensional frequency, $\eta$ is non dimensional "time" parameter (Farge, 1992). The continuous wavelet transform of a discrete sequence $x_{n}$ is defined as the convolution of $x n$ with a scaled and translated version of $\psi_{0}(\eta)$ (Grinsted et al., 2004; Jevrejeva et al., 2003; Torrence and Compo, 1998; Paschmann and Daly, 1998):

$W_{n}(\mathrm{~s})=\sum_{n^{\prime}}^{N-1} x_{n^{\prime}} \psi^{*}\left[\frac{\left(n^{\prime}-n\right) \delta t}{s}\right]$,

where the $\left(^{*}\right)$ indicates the complex conjugate, $\left|W_{n}(s)\right|^{2}$ the wavelet power spectrum of $\left\{x_{n}\right\}$. By varying the wavelet scale $\mathrm{s}$ and translating along the localized time index n, one can construct a picture showing both the amplitude of any features versus the scale and how this amplitude varies with time. The subscript 0 on $\psi$ has been dropped to indicate that this $\psi$ has also been normalized (Torrence and Compo, 1998). 


\section{$\underline{\text { Fourier Analysis }}$}

A power spectral density (PSD) signal analysis was also performed. The discrete Fourier transform definition of a discrete set $\mathrm{u}[\mathrm{j}]$ of $\mathrm{N}$ measurements: $\mathrm{j}=0,1, \ldots, \mathrm{N}-1$ :

$$
\tilde{u}[n]=\frac{1}{N} \sum_{j=0}^{N-1} u[j] \exp \left(\frac{2 \pi i n j}{N}\right) .
$$

Parseval's relation gives PSD in form:

$$
S_{u}[n]=\frac{2 N}{f_{s}}|\tilde{u}[n]|^{2}, n=0,1,2, \ldots . N / 2,
$$

where fs is a sampling frequency (Eriksson,2001; Paschmannand Daly, 1998). We illustrate below implementation of these methods to data obtained by Cluster-II mission spacecraft in terrestrial magnetotail.

\section{Implementation of Techniques for the Analysis of Turbulent Environment}

\section{Used Data}

For the analysis of the characteristics of turbulent processes in the tail of the Earth's magnetosphere, fluxgate magnetometer (FGM) measurements of 4 spacecraft from space mission "Cluster-II" with a sampling frequency of 22.5 $\mathrm{Hz}$ were used. The correct analysis of the magnetic field fluctuations, especially on small scale, cannot be done without high sampling frequency of experimental data. In this work we study spectral properties of current disruption (CD) event on August 12, 2014 using measurements of Cluster mission spacecraft, which were located in the magneto tail at geocentric distance 13-14 RE. Current disruption is the reduction of cross-tail current with changing of the tail magnetic field configuration from a stretched field lines to more dipolar. This phenomenon called dipolarization (DP) is accomplished with large-amplitude turbulent magnetic field fluctuations. The properties of the magnetic field fluctuations of this event were initially studied in Kronberg et al., 2017.

Changes in the magnetic field components $\mathrm{Bx}, \mathrm{By}, \mathrm{Bz}$ in GSM coordinate system and inclination angle $\theta$ (or latitude angle) defined as $\theta=\arctan \left(B_{z} / \sqrt{B_{x}^{2}+B_{y}^{2}}\right)$ for this event are shown in Figure 1. From the figure, it is seen that during the dipolarization, the fluctuations show higher variability of the parameters of the plasma (magnetic field).

High latitude values of inclination angle $\left(\theta\right.$ close to $\left.90^{\circ}\right)$ indicate location vicinity of spacecraft to the neutral sheet. Selection criteria for dipolarization, highlighted in work of Schmid et al. A statistical and event study of magnetotail, 
2011 comprise such requirements for latitude angle and $\mathrm{Bz}$ change: $\max (\theta)>$ 45, $\Delta \theta>10$, and $\max (\mathrm{Bz})-\min (\mathrm{Bz})>4 \mathrm{nTin} 3$-min time window. Jumps for Bz component are roughly $\sim 15 \mathrm{nT}$ from $\sim 10 \mathrm{nT}$ to $\sim 25 \mathrm{nT}$. Criteria in our case are satisfied. As it can be clearly seen in Figure 1 time interval of fluctuations for $\mathrm{Cl} 2$ (second panel) is shifted compared to interval of fluctuations for other satellites. Spacecraft $\mathrm{Cl} 1, \mathrm{Cl} 3, \mathrm{Cl} 4$ passed through dipolarization region approximately at 20:34:30-20:34:31 UT unlike Cl2 passing at 20:35:30 UT therefore time shift $\Delta \mathrm{T}$ is $60 \mathrm{~s}$. Location of $\mathrm{Cl} 1$ at 20:34:30 UT and $\mathrm{Cl} 2$ at 20:35:30 UT (X,Y,Z)GSM $=(-13.11,-0.79,1.75)$ RE and $(X, Y, Z) G S M=(-$ 13.66,-1.66,1.37) RE respectively (X,Y,Z)GSM (forCl1) - (X,Y,Z)GSM(for $\mathrm{Cl} 2)=(0.55,0.87,0.38) \mathrm{RE}$, so distance between $\mathrm{Cl} 1$ and $\mathrm{Cl} 2$ along $\mathrm{X}$-axis of GSM is $0.55 \mathrm{RE}$. Thereby there is a place to be dipolarization tail ward progression with an apparent speed $0.55 \mathrm{RE} \cdot \mathrm{min}^{-1}$. That is close to value $0.50 \mathrm{RE} \cdot \mathrm{min}^{-1}$ (Baumjohann, 1999; Kronberg et al., 2017). Also distinguishing property of this event is that large amplitude fluctuations were starting before depolarization when $\mathrm{B}$ and $\mathrm{Bz}$ magnitudes become approximately equal in the same time when Bx, By close to zero.

Figure 1a. Magnetic Field Measurements of Cluster Mission in GSM Coordinates with Inclination Angle during Dipolarization Event on August 12, 2014 (Cluster-1, Cluster-2)

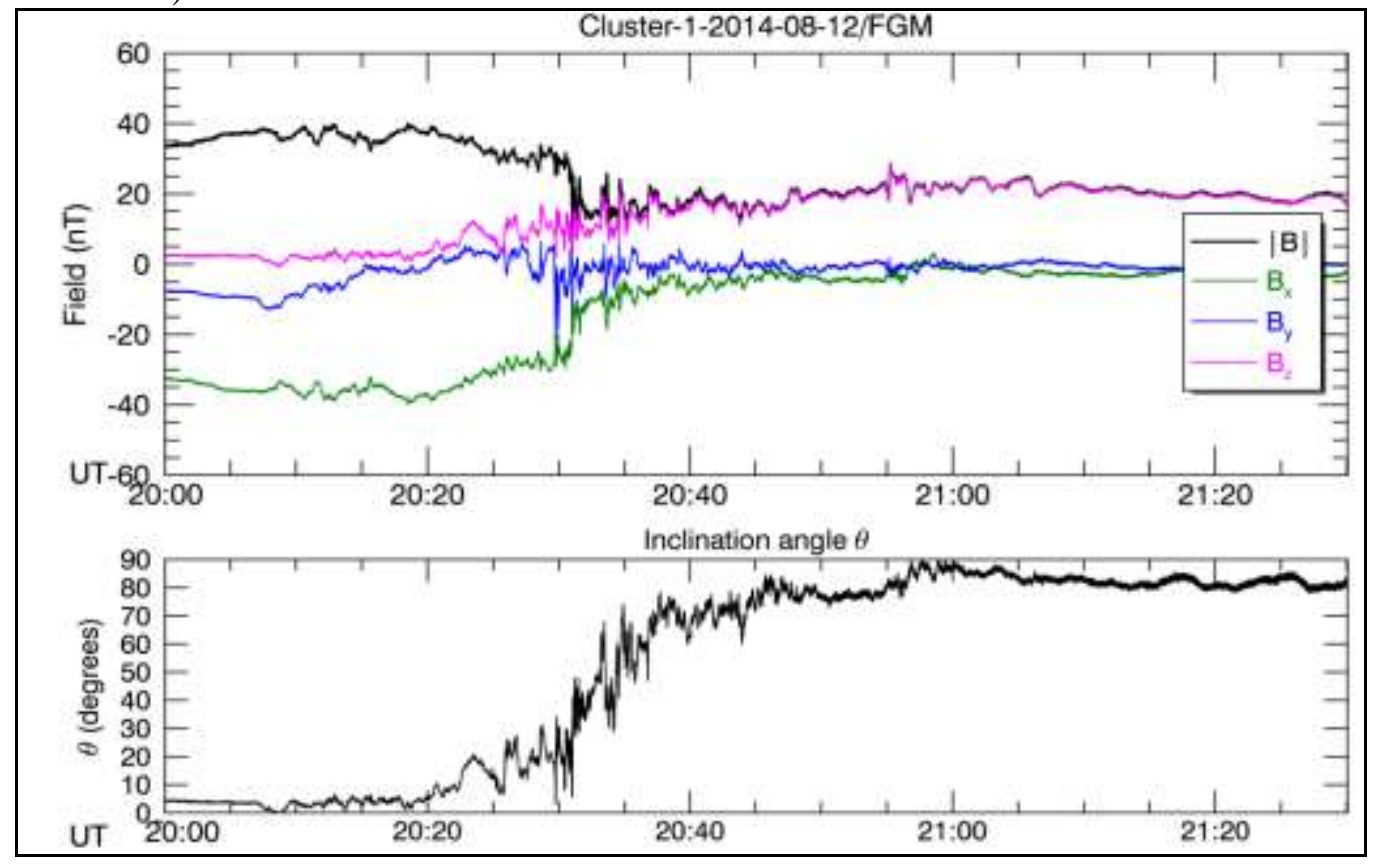




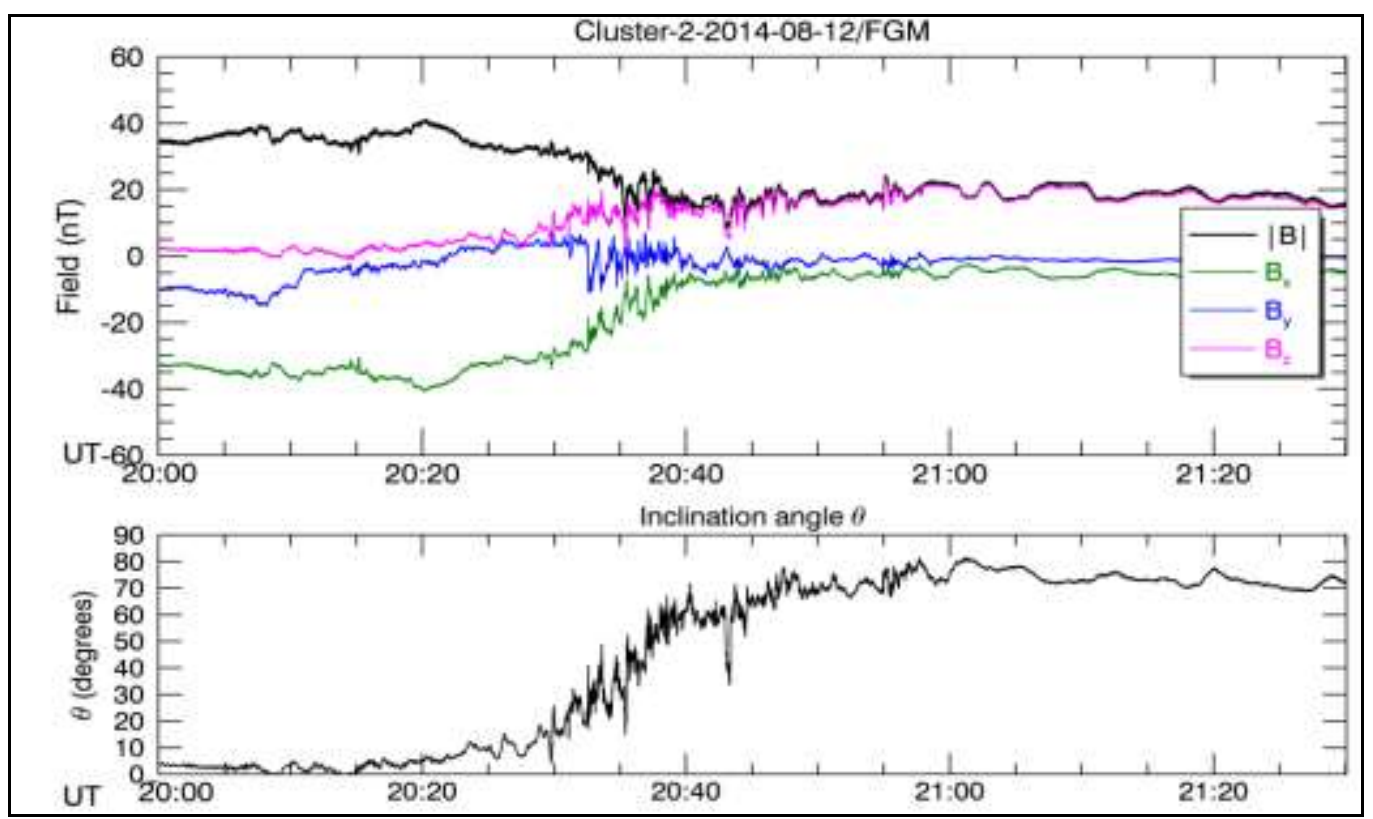

Figure 1b. Magnetic Field Measurements of Cluster Mission in GSM Coordinates with Inclination Angle during Dipolarization Event on August 12, 2014 (Cluster-3, Cluster-4)

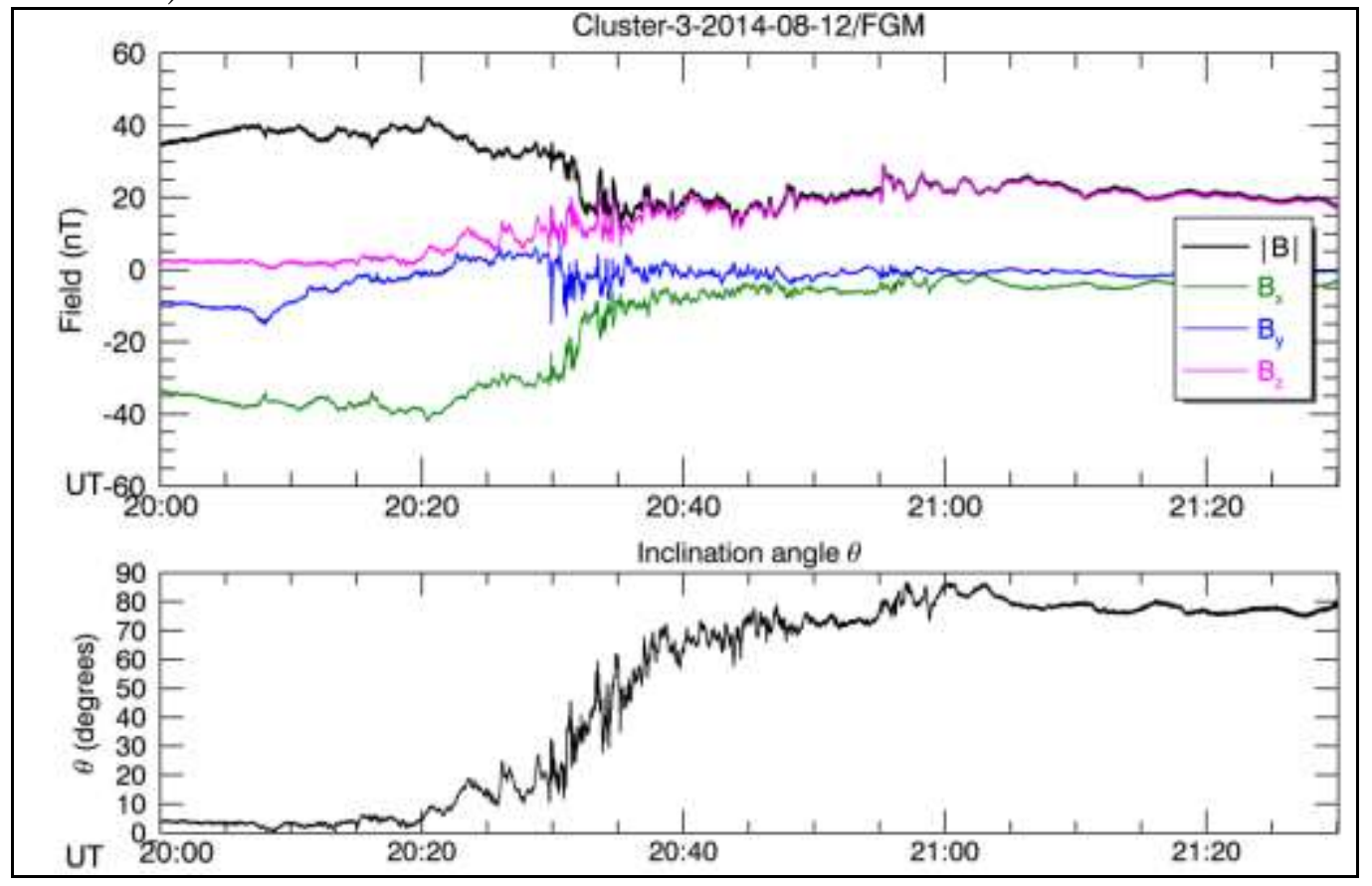




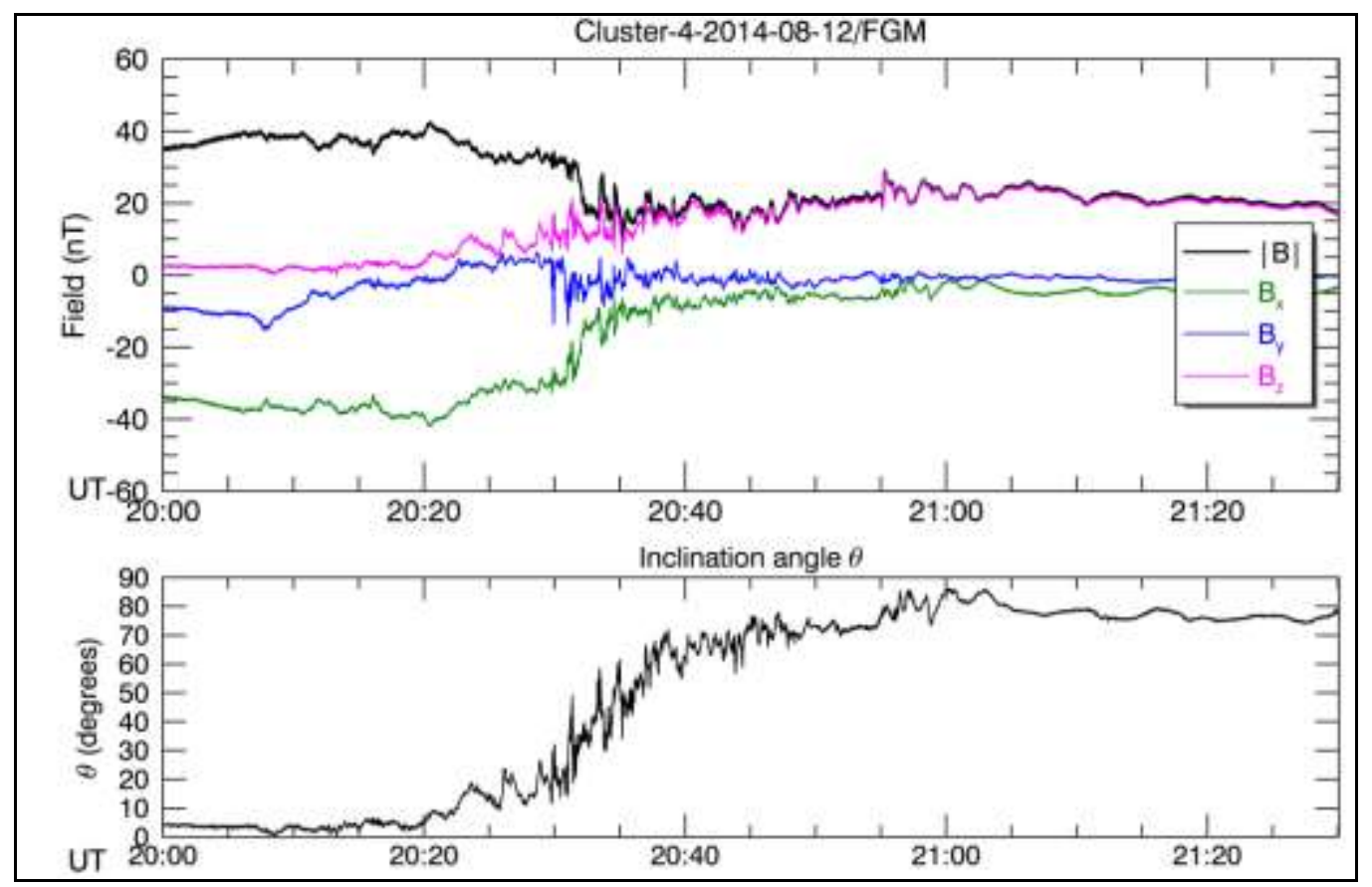

Results of Statistical Analysis of Magnetic Field Fluctuations in the Terrestrial Magnetotail

For studying the features of the probability distribution function of magnetic field fluctuations, time lags were chosen a multiple of sample time that is $\tau \min =0.0445 \mathrm{sec}$. The statistical properties of the absolute value of the magnetic field variations $d B=B(t+\tau)-B(t)$ in the Earth's magneto tail were analyzed. Figure 2 shows these distributions for different time lags before, during dipolarization event. It is clearly seen that during dipolarization event probability distribution tails become more significant. The shape is widened at least four times. 
Figure 2. The Example of the Probability Distribution Functions (PDFs) of the Evolution of the Magnetic Field Fluctuations at Different Timescales (1- $\tau=0.0445$

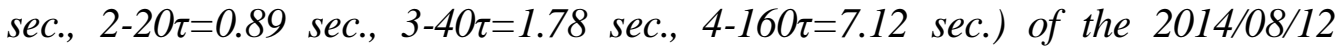
Dipolarization Event: a)Before, b)During Dipolarization Event

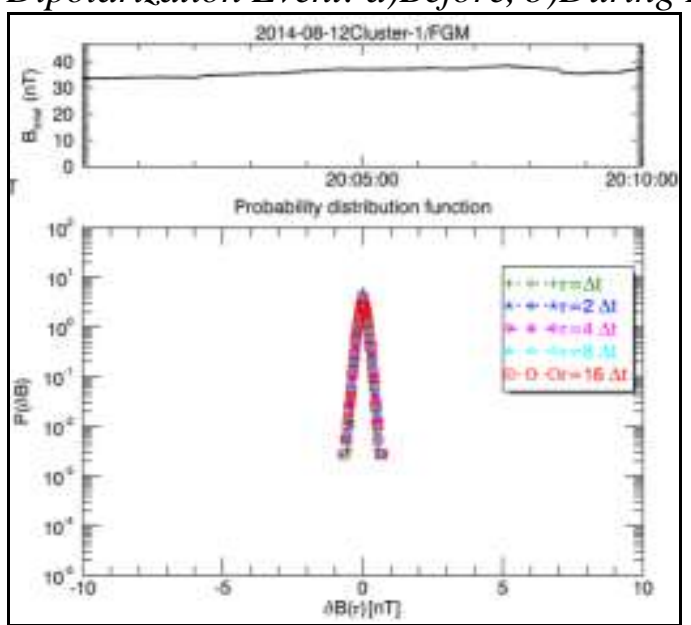

a)

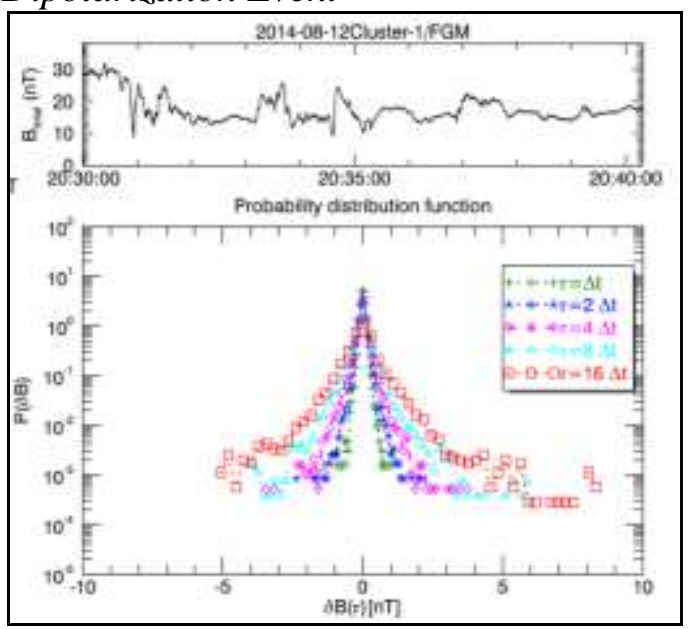

b)

To determine the type of turbulent processes observed in the plasma of the magnetotail, an analysis of the peculiarities of the structure functions (moments of the probability distibution function) of different orders $q$ according to the time interval $\tau$ for a series of data $B(t)$ has been carried out. Good discreteness of measurements allowed characterizing heterogeneity properties on small scale of the process. In this case, the structure function was determined by the relation (Kozak, 2010; Kozak et al., 2014; Kozak et al., 2017):

$S_{q}(\tau)=\left\langle|B(t+\tau)-B(t)|^{q}\right\rangle \sim \tau^{\zeta(q)}$,

where $\langle\ldots\rangle$ means the averaging of experimental data over time.

In view of the significant level of magnetic field fluctuations and the presence of flow boundaries, the analysis of extended self-similarity was carried out not only when compared with homogeneous models of turbulent processes (K41 and IK), but with a log-Poisson model of turbulence, which, depending on the values of the parameters characterizing the intermittence $\beta$ and the form dissipative structures $\Delta$, various types of available turbulent processes can be described. In this case, for the log-Poisson isotropic 3D turbulent cascade model (log-Poisson model with She and Leveque parameters), we have the dependence of the scaling $\zeta(\mathrm{q}):{ }(q)=q / 9+2\left[1-(2 / 3)^{q / 3}\right]$.

In practice, the property of generalized self-similarity can improve the accuracy of the definition $\zeta(q)$ when analyzing experimental data. Structure function scaling normalized to a scaling of 3rd order, $\zeta(\mathrm{q}) / \zeta(3)$ can be calculated from the graphic slope in a logarithmic scale (Figure 3a) (comparison of experimental data with the Kolmogorov model of turbulence K41). For comparison with two-dimensional Iroshnykov-Kraichnan model the relation $\zeta(\mathrm{q}) / \zeta(4)$ should be found (Figure $3 \mathrm{~b}$ ). 
Figure 3. Demonstration of Extended Self-similarity in Magnetic Field Turbulent Fluctuations during 2014/08/12 Dipolarization Event for Cl3: a) Scaling Estimation, $\zeta(q) / \zeta(3)$ b)Scaling Estimation $\zeta(q) / \zeta(4)$
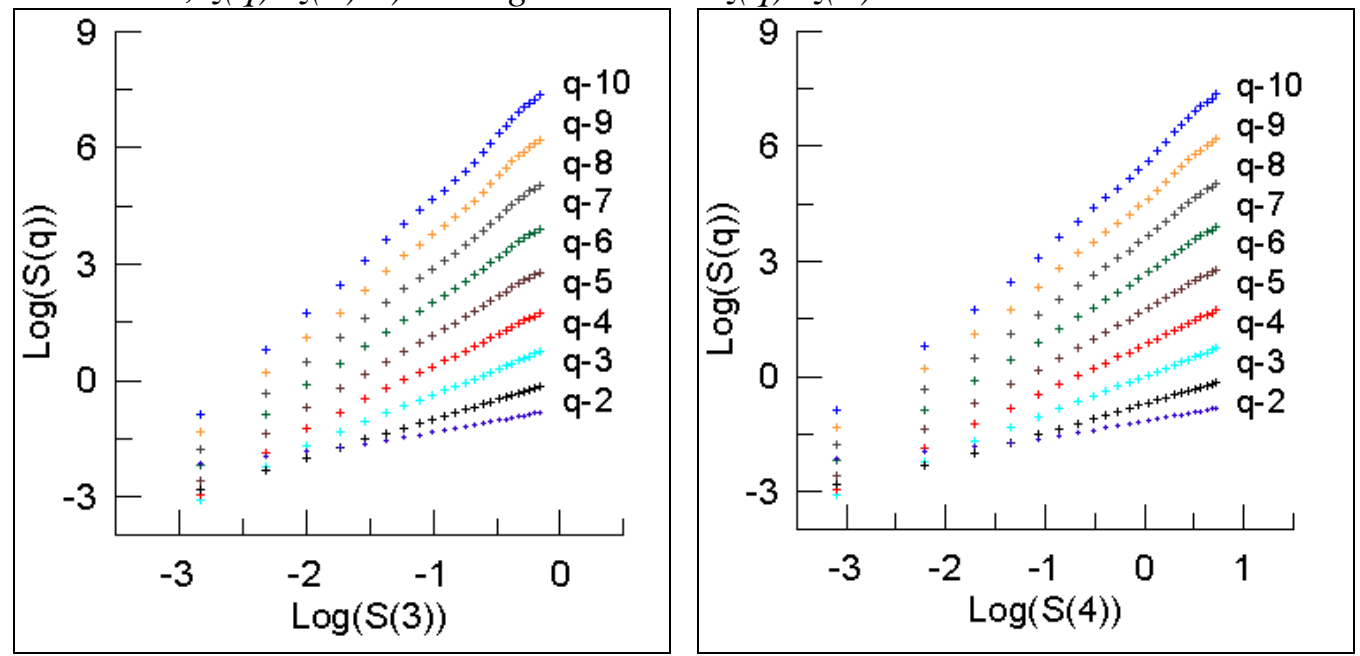

a)

b)

Figure 4. Structure Functions of Different Orders during 2014/08/12 Dipolarization Event by Cluster1 Measurements

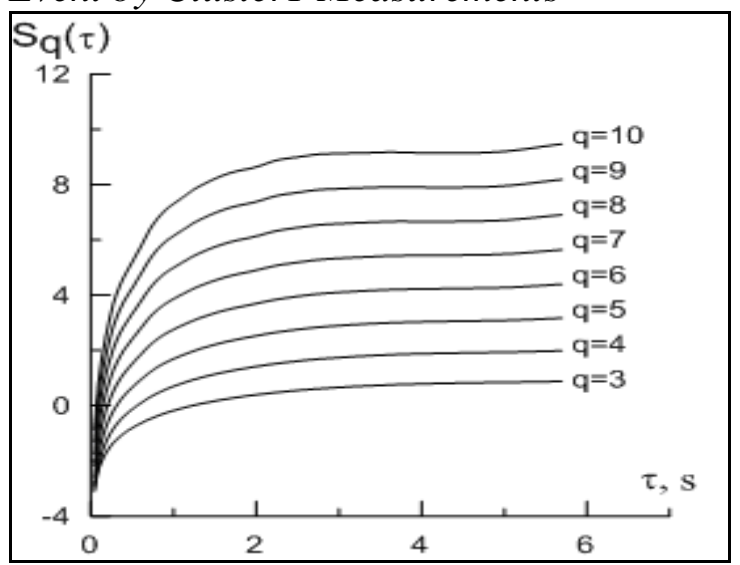

From Figure 4, it is clear that the power dependence of the type $\operatorname{Sq}(\tau) \sim \tau^{\zeta(\mathrm{q})}$ (i.e., self-similarity - the presence of linear dependence) is observed in limited timescale range up to $\sim 1$ second. This interval corresponds to the inertial range considered in the classical models of isotropic developed turbulence (K41, etc). In our event, this range is close to the ion-cyclotron frequency.

Given this, as well as the availability of data with high discreteness of measurements, we managed to conduct an ESS analysis to examine the properties of turbulent processes on small scales - up to $1 \mathrm{sec}$ (usually the discreteness of even satellite measurements does not allow doing similar analysis).

The results of scaling the moments of the probability distribution function for different orders of ${ }^{q}$ in the analysis of small-scale turbulence and comparison 
them with the Kolmogorov model, the two-dimensional model of IroshnikovKraichnan, and the isotropic log-Poisson model are presented in Figures 5 and 6.

Figure 5. Ratio of the Exponential Value of Structure Function of q-th Order to the 3rd One

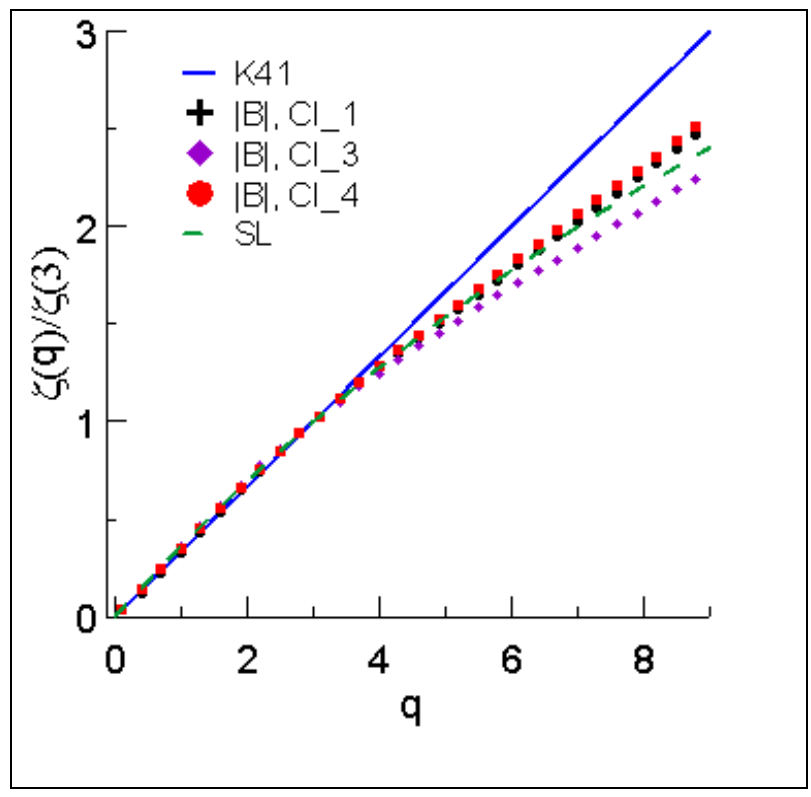

Results of ESS from Figure 5 give values of log-Poisson model parameters $\beta$ and $\Delta v$ during dipolarization of magnetic field configuration (see Table 1) and possibility of their application to find features of turbulent plasma transfer. Under such approach general diffusion coefficient D depends on structure function scaling $\zeta(q)$ in next way (Chechkin et al., 2002):

$D \propto \tau^{R}, R(q)=q-(3 q)$.

This kind of scaling is used for transport estimation in statistically inhomogeneous medium. In general, scaling $\mathrm{R}$ is governed by fractal characteristic of this medium. Estimated values of $\mathrm{R}$ lies in range $0.27 \div 0.66$. Particle displacements depend on time, therefore the following relation is valid:

$\left\langle\delta \mathrm{x}^{2}\right\rangle \propto \mathrm{D} \tau \propto \tau^{\delta}$

With exponent $\delta \propto 1+\mathrm{R} \approx 1.27 \div 1.66>1$, that signifies an existence of superdiffusion state. 
Figure 6. Ratio of the Exponential Value of Structure Function of q-th Order to the 4rd One

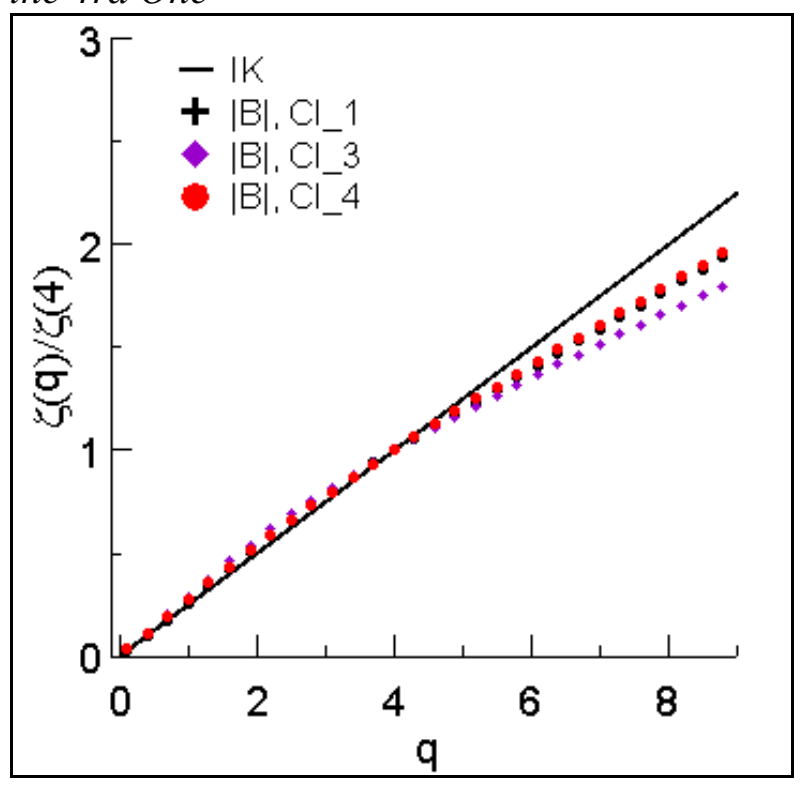

Table 1. Estimation of Log-Poisson Scaling Parameters

\begin{tabular}{|l|c|c|c|}
\hline Satelite & $\beta$ & $\Delta$ & $\mathrm{R}=\Delta(1 / \beta-1)$ \\
\hline Cluster-1 $\left(\mathrm{Cl} \_1\right)$ & $0.53 \pm 0.012$ & $0.49 \pm 0.011$ & 0.43 \\
\hline Cluster-2 $\left(\mathrm{Cl} \_2\right)$ & $0.32 \pm 0.018$ & $0.31 \pm 0.01$ & 0.66 \\
\hline Cluster-3 $(\mathrm{Cl}$ 3) & $0.74 \pm 0.009$ & $0.56 \pm 0.012$ & 0.19 \\
\hline Cluster-4 $\left(\mathrm{Cl} \_4\right)$ & $0.71 \pm 0.015$ & $0.66 \pm 0.012$ & 0.27 \\
\hline
\end{tabular}

Results of Spectral Analysis of Magnetic Field Fluctuations

The results of the conducted spectral analysis show the presence of different characteristic scales (see Figure7). Spectral density has kink approximately at 1 $\mathrm{Hz}$ and evident artificial frequency at $0.25 \mathrm{~Hz}$ that corresponds to satellite spin frequency. Spectral indices in ranges $0.01-1 \mathrm{~Hz}$ are given below in Table 2 . During dipolarization event shape of spectrum becomes steeper. Before and after DP spectral index is close to 2 .

Table 2. Spectral Indices from PSD Analysis

\begin{tabular}{|l|l|l|l|}
\hline Satelite & $\begin{array}{l}\text { Before DP } \\
(20: 00-20: 10 \text { UT })\end{array}$ & $\begin{array}{l}\text { During DP } \\
(20: 30-20: 40 \text { UT })\end{array}$ & $\begin{array}{l}\text { After DP } \\
(21: 00-22: 00 ~ U T)\end{array}$ \\
\hline Cluster-1 $\left(\mathrm{Cl} \_1\right)$ & $-1.931 \pm 0.041$ & $-2.418 \pm 0.059$ & $-2.118 \pm 0.020$ \\
\hline Cluster-2 $(\mathrm{Cl} 22)$ & $-2.084 \pm 0.043$ & $-2.263 \pm 0.053$ & $-1.942 \pm 0.020$ \\
\hline Cluster-3 $\left(\mathrm{Cl} \_3\right)$ & $-2.076 \pm 0.031$ & $-2.400 \pm 0.054$ & $-2.061 \pm 0.018$ \\
\hline Cluster-4 $\left(\mathrm{Cl} \_4\right)$ & $-2.023 \pm 0.035$ & $-2.245 \pm 0.056$ & $-2.099 \pm 0.019$ \\
\hline
\end{tabular}


Vol. 5, No. 2 Kozak et al.: Characteristics of the Turbulence Processes in the...

Figure 7. The Example of the PSD (Power Spectral Density) with Spectral Slopes (0.01 Hz-1Hz, $1 \mathrm{~Hz}-11.25 \mathrm{~Hz})$ Before, During and After 2014/08/12 Dipolarization Event for Cluster-1

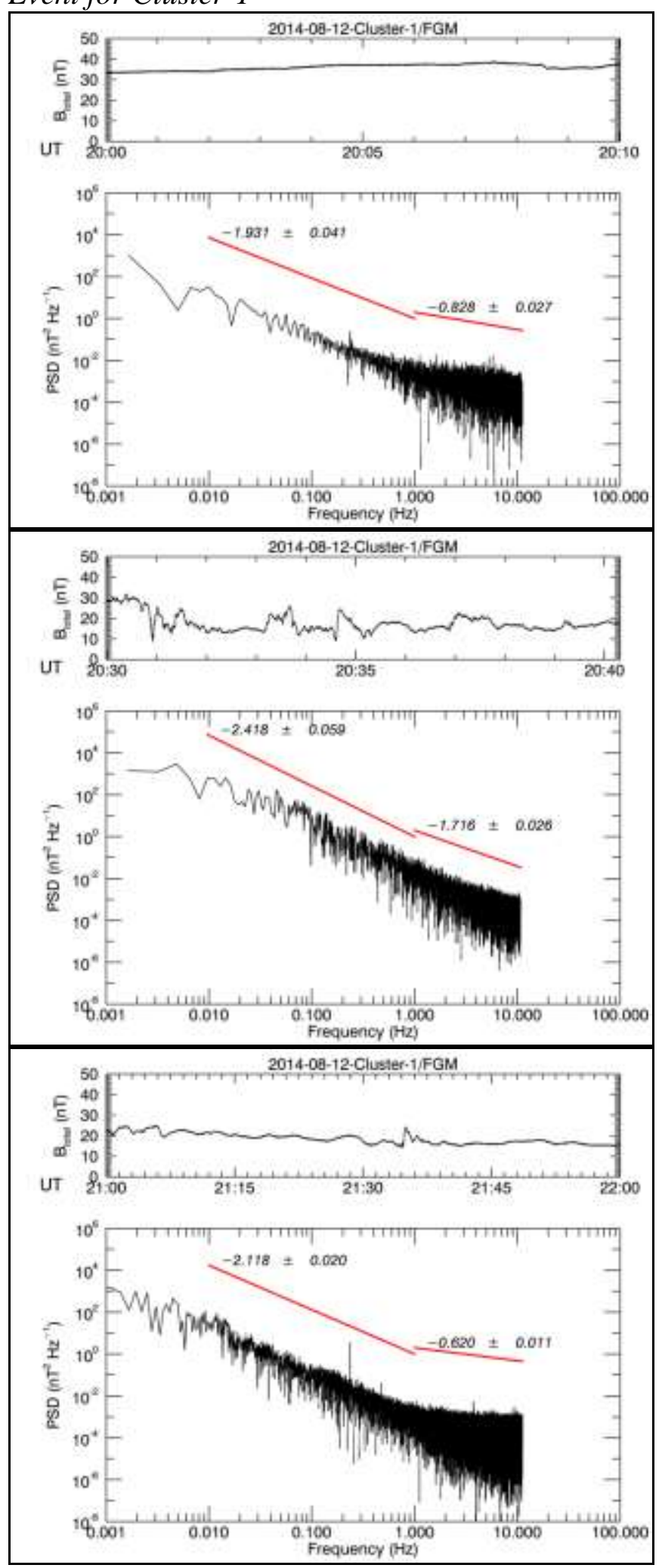


Figure 8shows the results of spectral analysis by usage of continuous wavelet transform with Morlet mother wavelet over an interval 20:25-20:40 UT. The temporal resolution of the magnetic field data is $0.0455 \mathrm{~s}$, thus upper frequency - Nyquist frequency is approximately $11.25 \mathrm{~Hz}$. The frequency domain is divided into 10 octaves, each with 8 suboctaves. Figure 8 show the wavelet analysis for the magnetic field module for all Cluster spacecraft.Let us examine frequency components which are presented in signals. For all spacecraft during dipolarization event inverse cascade feature is observed, even before formal onset of DP as for $\mathrm{Cl} 1$ where there was the inverse cascade feature at 20:31-20:32 UT from 0.08 to $0.04 \mathrm{~Hz}$ i.e. reduction of wave frequency component from high level of frequency to low. For all satellites except $\mathrm{Cl} 2$ there was a prolonged low-frequency component of middle intensity $\sim 8 \mathrm{nT}$ at $0.02-0.03 \mathrm{~Hz}$, which exists at 20:25-20:30 UT that is before the dipolarization, possible explanation is an existence of pc4 oscillations. Simultaneously for Cl1 intensifications of wave power at $0.1 \mathrm{~Hz}, 0.05 \mathrm{~Hz}, 0.03 \mathrm{~Hz}, 0.015 \mathrm{~Hz}$ is seen at 20:33-20:35 UT. High enhancements in the wavelet intensity for $\mathrm{Cl} 2$ appeared preferentially during DP and intense $(\sim 15 \mathrm{nT})$ but not plently broad in frequency domain (gradual decreasing from 0.025 to $0.015 \mathrm{~Hz}$ ) during 3-min interval 20:35-20:38 UT. Also there were components with inverse cascade features in frequency range of $0.07-0.2 \mathrm{~Hz}$. Wavelet activity for $\mathrm{Cl} 3$ and $\mathrm{Cl} 4$ satellites has similar appearance because of closeness of their mutual relative location. Starting from 20:29 UT the wave power was enhanced at high frequency of $0.2-0.5 \mathrm{~Hz}$ for $\mathrm{Cl} 3$ and $\mathrm{Cl} 4$ measurements. After mutual separation from basic preonset low component $0.015 \mathrm{~Hz}$ at 20:33 UT merging of two frequency components with $0.015 \mathrm{~Hz}$ and $0.03 \mathrm{~Hz}$ to $0.02 \mathrm{~Hz}$ was observed at 20:35:30 UT. Inverse cascades were distinctly present e.g. from 0.07 to 0.05 $\mathrm{Hz}$ at 20:34:30 UT. Quite localized in time (duration $~ 30 \mathrm{~s}$ ) direct cascade from 0.08 to $0.15 \mathrm{~Hz}$ at 20:34 UT was observed unlike for Cl4 wavelet spectrogram at this time inverse cascade from 0.2 to $0.08 \mathrm{~Hz}$ and separately existing $0.15 \mathrm{~Hz}$ were presented.

The results of the wavelet analysis indicate the existence of cascading processes - mostly inverse cascade processes, which indicate the multiscale nature of the magnetosphere's dynamics.

Wavelet transform reveal a constant in time frequency component $0.25 \mathrm{~Hz}$ (see Figure 9), but with different intensity along all signal, comparing with Figure 8. This frequency has an artificial origin corresponding to spin tone of Cluster satellites 15 rotations per minute. Why is spin tone not clearly equal in wavelet intensity along time domain especially considering its weakness during DP? We try to give possibly satisfactory explanation. First of all intense magnetic field fluctuations during DP "conquer" weak in flux spin tone, unlike after DP. The second cause is possible due to continuos change of angle between spin axis and magnetic field. 
Figure 8a. Wavelet Analysis of the Magnetic Field Module during 2014/08/12 Dipolarization Event (Cluster-1, Cluster-2)
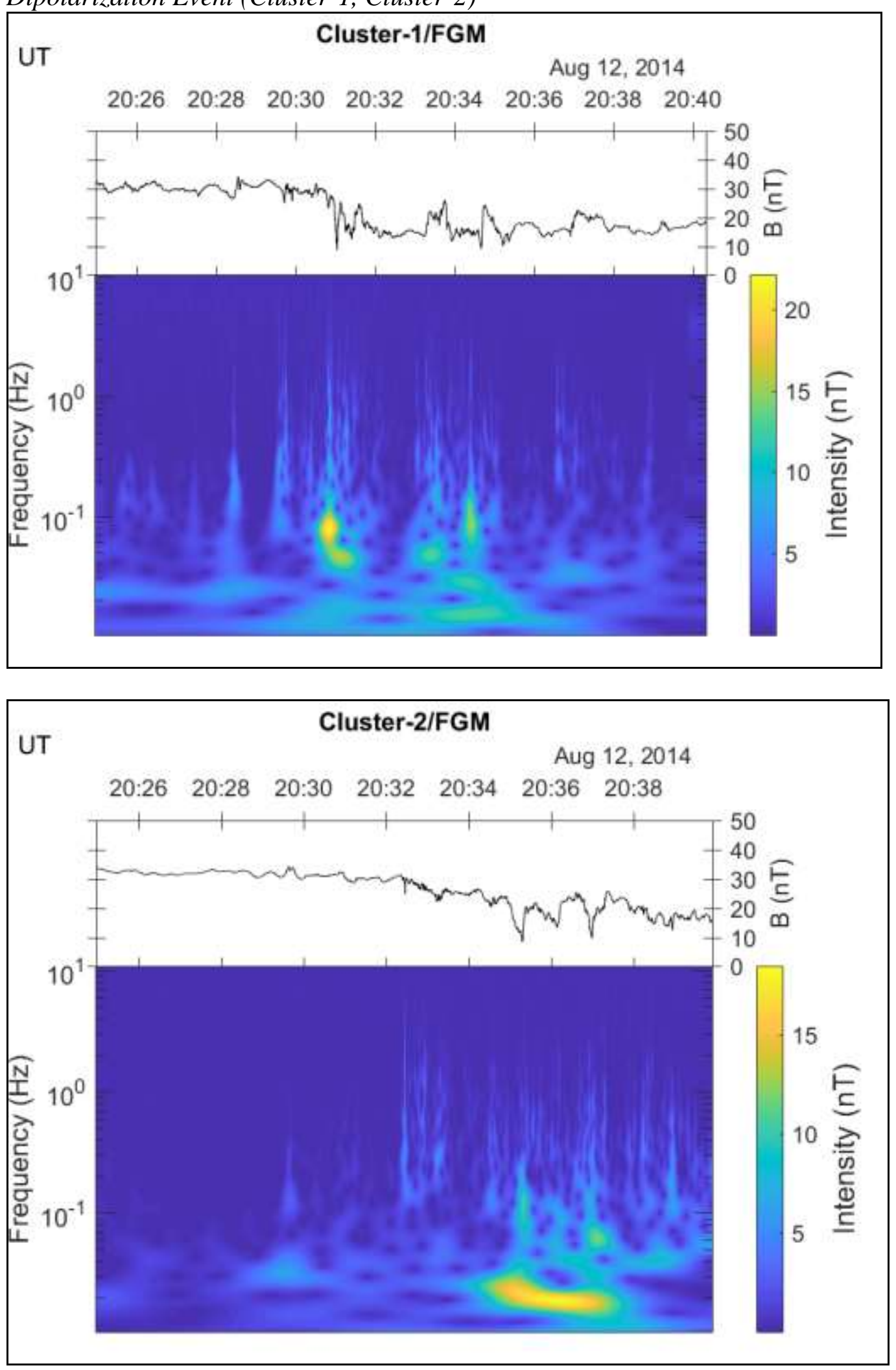
Figure 8b. Wavelet Analysis of the Magnetic Field Module during 2014/08/12 Dipolarization Event (Cluster-1, Cluster-2)
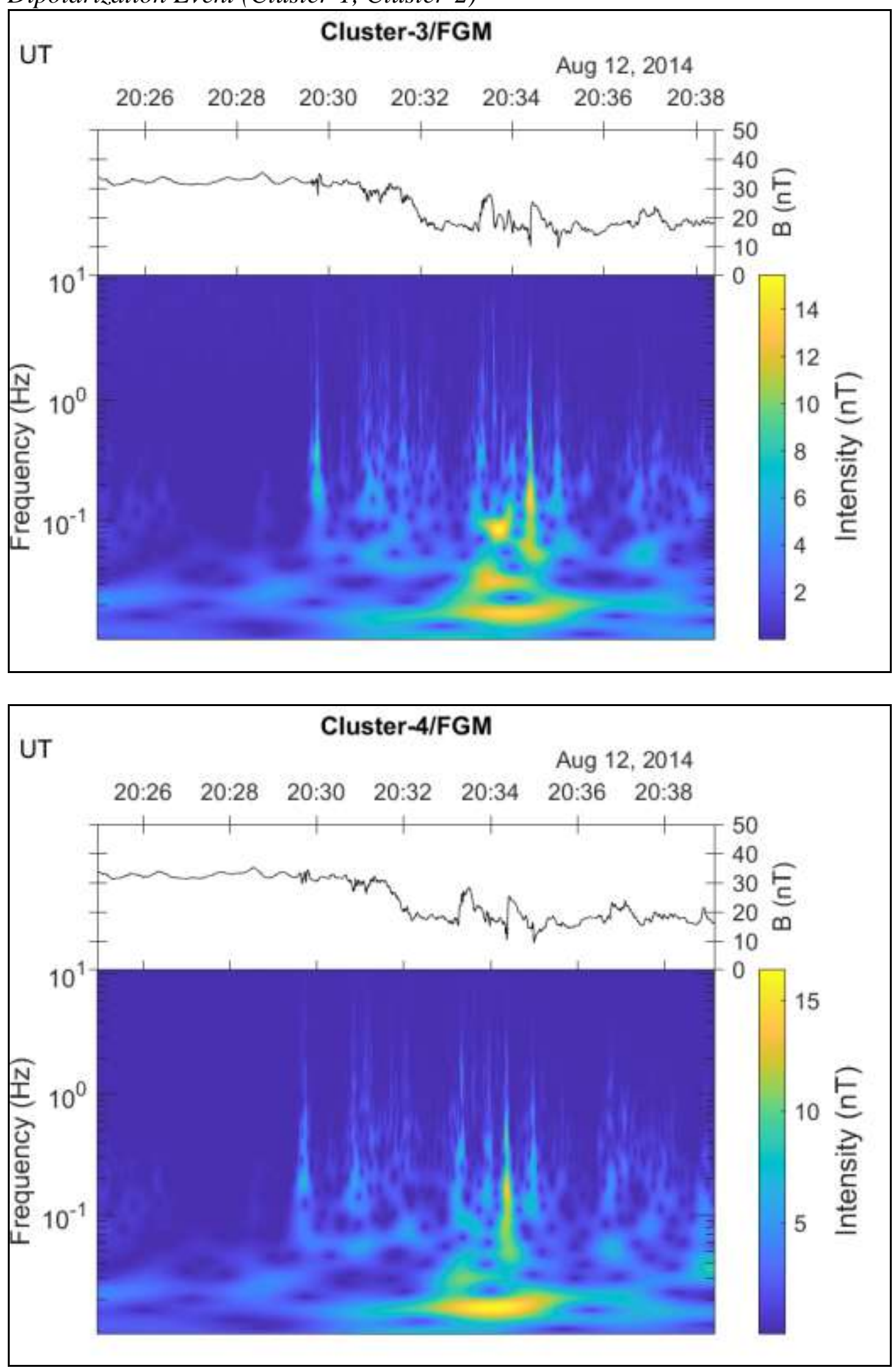
Figure 9. Wavelet Analysis of the Magnetic Field Module after 2014/08/12 Dipolarization Event with Evident Artificial Frequency Component $0.25 \mathrm{~Hz}$

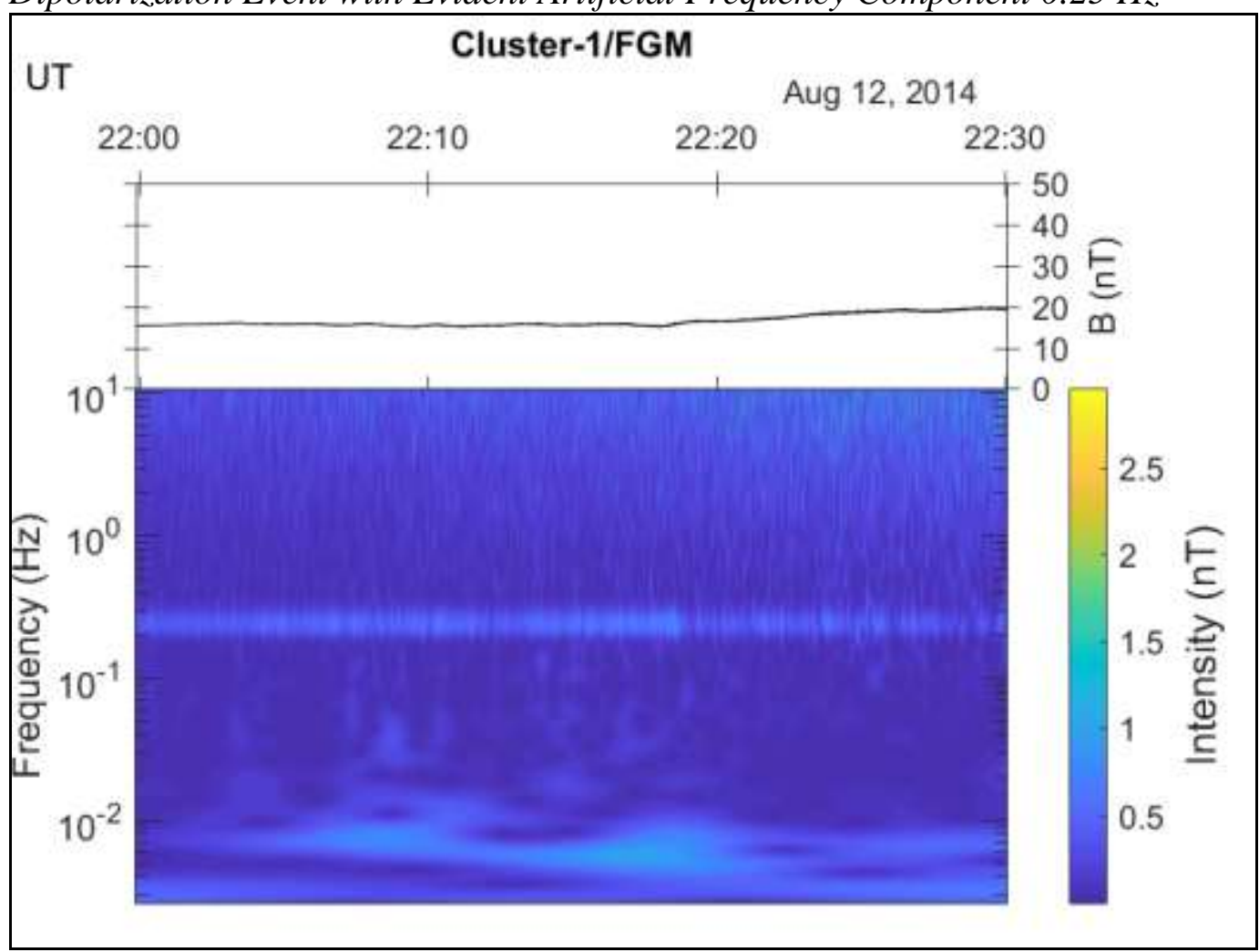

\section{Conclusions}

For all the measurements considered during the dipolarization of the magnetic field, the distribution function of the magnetic field fluctuations is substantially widening than at moments during DP. Power law tails indicate non-Gaussian process statistics, as well as the energy excess of large-scale perturbations generated by the source.

The transition of the statistics from Gaussian to non-Gaussian is possibly due to relaxation process changing from simple to complex, involving an increase of the range of interaction and correlation that reveals itself as fast processes. Furthermore, non-MHD nature of the DP phenomenon involving fast processes in high- $\beta$ non collisional plasmas handles the occurrence of fast relaxation process at frequencies above the characteristic ion cyclotron frequency.

Comparing the high order structure functions of magnetic field fluctuations during dipolarization event from the models of Kolmogorov, Kraichnan and with $\mathrm{c}$ $3 \mathrm{D}$ isotropic log-Poisson model of turbulence with the parameters She and Leveque, we obtain that these turbulent processes cannot be described by isotropic homogeneous models of turbulent processes.

Using the intermittency and singularity coefficients found in the ESS analysis for investigations of turbulent processes, the power dependence of the generalized 
diffusion coefficient on the scale is obtained (the scaling varies within range of 0.19-0.66). This indicates the presence of super diffusion processes.

One of the important results is the essential changing of power spectral index before and during the dipolarization of the magnetic field lines. Before the dipolarization, the power spectral index is varying from $-1.931 \pm 0.041$ to $2.084 \pm 0.043(\sim-5 / 3$ corresponds to the Kolmogorov model of turbulent processes). For the moments of the dipolarization the power spectral index is from $-2.245 \pm 0.056$ to $2.418 \pm 0.059(\sim-7 / 3$ - the electron-magnetohydrodynamics (EMHD) turbulence). The EMHD theory describe the behavior of high- $\beta$ plasmas at timescales shorter than the ion cyclotron period and spatial scales smaller than the ion inertial length, where most of the dynamics of the plasma is governed by electrons (Biskamp et al., 1996; Boldyrev, 2006).

The wavelet analysis showed the presence of both direct and reverse cascade processes, as well as the presence of PC pulsations. The presence of reverse cascade processes points to the possibility of self-organization processes.

Thus, during the dipolarization, the large-scale and multi-fractal disturbances of the magnetic field are observed.

The set of different methods and approaches to study the characteristics of the turbulent medium in the tail of the Earth's magnetosphere showed a good match and mutual complementation.

\section{Acknowledgments}

The work is done in the frame of the grant Az. 90312 from the Volkswagen Foundation («VW-Stiftung»).

\section{References}

Barenblatt, G.I.2004. Turbulent boundary layers at very large Reynolds numbers. Russian Mathematical Surveyes.59, 45-62.

Baumjohann, W., Hesse, M., Kokubun, S., Mukai, T., Nagai, T., and Petrukovich, A. 1999. Substorm Dipolarization and Recovery. J. Geophys. Res.104, A11, 2499525000.

Benzi, R., Ciliberto, S., Tripiccione, R., Baudet, C., Massaioli, F., and Succi, S. 1993. Extended self - similarity in turbulent flows. Phys. Rev. E.48, R29-R32.

Biskamp, D., Schwarz, E., and Drake, J. F. 1996. Two dimensional electron magnetohy-drodynamic turbulence.Phys. Rev. Lett.76, 8, 1264-1267.

Boldyrev, S. 2006 Spectrum of Magnetohydrodynamic Turbulence. Phys. Rev. Lett. 96.id. 115002.

Chang, T. 1992. Low dimensional behavior and symmetry breaking of stochastic systems near critically - can these effects be observed on space and in the laboratory? IEEE Transactions on Plasma Science.20, p. 691.

Chechkin, A. V., Gorenflo, R., and Sokolov, I. M. 2002. Retarding subdiffusion and accelerating superdiffusion governed by distributed-order fractional diffusion equations. Phys. Rev. E. 66, 4, - id.046129. 
Consolini, G., and Lui, A. T. Y. 2000.Symmetry Breaking and Nonlinear Wave-Wave Interaction in Current Disruption: Possible Evidence for a Phase Transition. American Geophysical Union. Washington, DC,p. 395.

Consolini, G., Kretzschmar, M., Lui, A.T.Y., Zimbardo, G., and Macek, W.M. 2005. On the magnetic field fluctuations during magnetospherictail current disruption: A statistical approach. J. Geophys. Res.110, A07202.

Dubrulle, B. 1994. Intermittency in fully developed turbulence: Log - Poisson statistics and generalized scale covariance. Phys. Rev. Lett.73, 959-962.

Eriksson, A.I. 2001. Spectral Analysis, Reprinted from Analysis Methods for MultiSpacecraft Data Gotz Paschmann and Patrick W. Daly (Eds.), ISSI Scientific Report SR-001 (Electronic edition 1.1).

Farge, M. 1992. Wavelet transforms and their applications to turbulence. Annu.Rev. Fluid Mech. 24, 395-457.

Frik, P.G. 1999. The turbulence: models and approaches. Perm's State Tech. Univ. Part II, p. 139.

Frisch, U. 1998. Turbulence: The Legacy of A. N. Kolmogorov. Moscow: Phasis. p. 343.

Gledzer, E. B. 2005. Dissipation and intermittency in the framework of hydrodynamic approximations. Izvestiya. Atmospheric and Oceab Physics41, 6, 667-683.

Grigorenko, E. E., Kronberg, E. A., Daly, P. W., Ganushkina, N. Y., Lavraud, B., Sauvaud, J.-A., and Zelenyi, L. M. 2016. Origin of low proton-to-electron temperature ratio in the Earth's plasma sheet, J. Geophys. Res. Space Physics, 121, doi:10.1002/2016JA02 2874.

Grigorenko, E. E., Dubyagin, S., Malykhin, A. Y., Khotyaintsev, Y. V., Kronberg, E. A., Lavraud, B., and Ganushkina, N. Y. 2018. Intense current structures observed at electron kinetic scales in the near-Earth magnetotail during dipolarization and substorm current wedge formation. Geophysical Research Letters, 45. https://doi.org/10.1002/ 2017GL076303.

Grinsted, A., Moore, J., and Jevrejeva, S. 2004. Application of the cross wavelet transform and wavelet coherence to geophysical time series. Nonlinear Processes in Geophysics. 11, 5/6, 561-566.

Jevrejeva, S., Moore, J., and Grinsted, A. 2003. Influence of the Arctic Oscillation and El Nieno-Southern Oscillation (ENSO) on ice conditions in the Baltic Sea: The wavelet approach. J. Geophys. Res. 108, 4677-4708.

Kolmogorov, A.N. 1941. Local Structure Turbulence in Noncompressible Flow under High Reynolds Numbers.USSR Academy Report 30, 4, 299-303.

Kozak, L. V. 2010.Statistical analysis of turbulent processes in the Earth's magnetosphere by Interbol satellite measurements.Space science and technology.16, 1, 28-39.

Kozak, L. V., Pilipenko, V. A., Chugunova, O. M., and Kozak, P. N.2011. Statistical analysis of turbulence in the foreshock region and in the Earth's magnetosheath. Cosmic Research 49,3, 194-204.

Kozak, L. V., Savin, S. P., Budaev, V. P., Pilipenko, V. A., and Lezhen, L. A.2012. Character of turbulence in the boundary regions of the Earth's magnetosphere. Geomagnetism and Aeronomy.52, 4. 445-455.

Kozak, L., Lui, A., and Savin, S. 2014.Statistical analysis of the magnetic field measurements. Odessa Astronomical Publications. 26/2,268-271.

Kozak, L. V., Prokhorenkov, A. S., and Savin, S. P. 2015. Statistical analysis of the magnetic fluctuations in boundary layers of Earth's magnetosphere. Advances in Space Research 56, 10,2091-2096.

Kozak, L. V. Lui, A. T. Y. Kronberg, E. A., and Prokhorenkov, A. S. 2017. Turbulent processes in Earth's magnetosheath by Cluster mission measurements. Journal of Atmospheric and Solar-Terrestrial Physics.154, P.115-126. 
Kraichnan, R. H. 1959. The structure of isotropic turbulence at very high Reynolds numbers. J. Fluid Mech. 5,497-543.

Kraichnan, R. H. 1970.Convergents to turbulence functions. J. Fluid Mech.41.189-217.

Kronberg, E. A., Grigorenko, E. E., Turner, D. L., Daly, P. W., Khotyaintsev, Y., and Kozak, L. 2017. Comparing and contrasting dispersionless injections at geosynchronous orbit during a substorm event. J. Geophys.Res.Space Physics, 122, 3055-3072, doi: 10.1002/2016JA023551.

Malykhin, A.Yu., Grigorenko, E.E., Kronberg, E.A., Koleva, R., Ganushkina, N. Yu., Kozak, L., and Daly, P.W. 2018. Contrasting dynamics of electrons and protons in the near-Earth plasma sheet during dipolarization.Annales Geophysicae.

Novikov, E. A., and Stewart, R. 1964.Intermittency of turbulence and spectrum of fluctuations in energy-disspation, Izv.Akad.Nauk.SSSR. Ser. Geofiz. 3. 408-413.

Paschmann, G., and Daly, P. W. 1998. Analysis Methods for Multi-Spacecraft Data. ESA Publications Division Keplerlaan.

She, Z., and Leveque,E. 1994.Universal scaling laws in fully developed turbulence. Phys. Rev. Lett.72. 336-339.

THOR, 2017. Exploring plasma energization in space turbulence. Assessment Study Report ESA/SRE, p.109.

Torrence, C., and Compo, G. P.A 1998. Practical Guide to Wavelet Analysis. Bull. Amer. Meteor. Soc. 79.61-78.

Zaslavsky, G. M., and Sagdeev, R. Z. 1988. Nonlinear Physics: From the Pendulum to Turbulence and Chaos. Moscow: Nauka, p. 368. 
